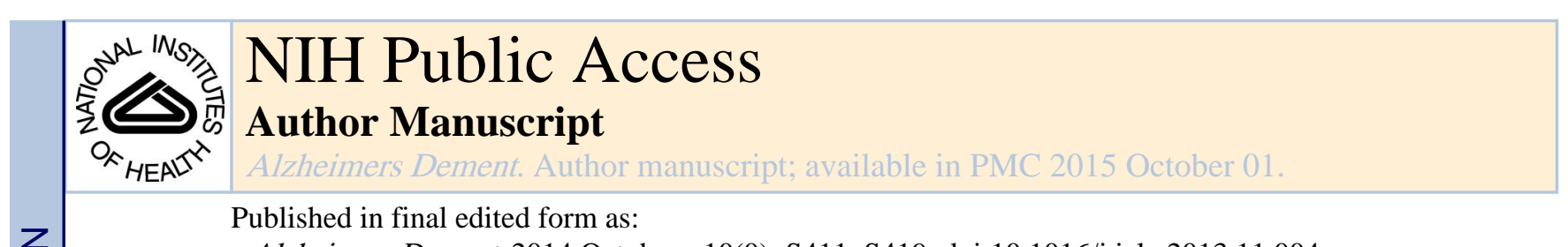

Alzheimers Dement. 2014 October ; 10(0): S411-S419. doi:10.1016/j.jalz.2013.11.004.

\title{
Lessons from a BACE inhibitor trial: Off-site but not off base
}

\author{
Debomoy K. Lahiria,b, ${ }^{,}$, Bryan Maloneya ${ }^{a}$ Justin M. Long, and Nigel H. Greig ${ }^{c}$ \\ aLaboratory of Molecular Neurogenetics, Department of Psychiatry, Institute of Psychiatric \\ Research, Indiana University School of Medicine, Indianapolis, 791 Union Drive, IN, USA \\ bDepartment of Medical and Molecular Genetics, Indiana University School of Medicine, \\ Indianapolis, 791 Union Drive, In, USA
}

'Laboratory of Translational Gerontology, Intramural Research Program, National Institute of Aging, NIH, Baltimore, MD, USA

\section{Abstract}

\begin{abstract}
Alzheimer's disease (AD) is characterized by neuritic plaque formation, which is primarily composed of a small filamentous protein called amyloid- $\beta$ peptide $(A \beta)$. The rate-limiting step in the production of $A \beta$ is the processing of $A \beta$ precursor protein (APP) by a $\beta$-site APP-cleaving enzyme (BACE1). Hence, BACE1 activity plausibly plays a significant role in the generation of potentially toxic $\mathrm{A} \beta$ within brain and the development of $\mathrm{AD}$, thereby making it an interesting drug target. A phase 2 trial of the promising LY2886721 inhibitor of BACE1 was suspended in June of 2013 by Eli Lilly and Company due to apparent liver toxicity. This outcome was apparently a surprise to the study's team, particularly since BACE1 knockout mice and mice treated with the drug did not show such liver toxicity. Lilly proposed that the problem was not due to LY2886721 anti-BACE1 activity. We offer an alternative hypothesis, whereby anti-BACE1 activity may induce apparent hepatotoxicity through inhibiting BACE1's processing of $\beta$ galactoside a-2,6-sialyltransferase I (STGal6 I). In knockout mice, paralogues, such as BACE2 or cathepsin D, could partially compensate. Furthermore, the short duration of animal studies and short lifespans of study animals could mask effects that would require several decades to accumulate in humans. Inhibition of hepatic BACE1 activity in middle-aged humans would produce effects not detectable in mice. In summary, we present a testable model to explain the offtarget effects of LY2886721 and highlight more broadly that so called off-target drug effects might actually represent off-site effects that are not necessarily off-target. Consideration of this concept in forthcoming drug design, screening and testing programs might prevent such failures in the future.
\end{abstract}

\footnotetext{
(C) 2013 Elsevier Inc. All rights reserved.

*Corresponding author: Indiana University School of Medicine, Institute of Psychiatric Research, Room 313, Indianapolis IN 46202, USA, 791 Union Drive Rm., Phone: 317-274-1362, Fax 317-274-1365, dlahiri@iupui.edu.

Publisher's Disclaimer: This is a PDF file of an unedited manuscript that has been accepted for publication. As a service to our customers we are providing this early version of the manuscript. The manuscript will undergo copyediting, typesetting, and review of the resulting proof before it is published in its final citable form. Please note that during the production process errors may be discovered which could affect the content, and all legal disclaimers that apply to the journal pertain.
} 


\section{Keywords}

Aging; animal model; brain disorder; CNS; dementia; demyelination; drug trial; liver damage; human studies; melatonin; neuronal death; sialylation; secretase; side effects; ROS

\section{Introduction}

Alzheimer's disease (AD) is the most common form of dementia in the elderly population of the United States [1]. AD is clinically characterized by a loss of cognition and memory, and this form of progressive dementia ultimately leads to changes in the patient's personality, incapacitation, and eventually loss of life. Major hallmarks of AD are deposits of amyloid plaques comprising mostly fibrillar amyloid beta $(\mathrm{A} \beta)$ peptide and neurofibrillary tangles comprising hyperphosphorylated tau [1]. Molecular mechanisms and environmental factors, including epigenetic and genetic, that underlie these pathological findings are still unclear [2-3]. Current drug therapies exist for AD but mostly aim to treat the symptoms of this devastating disease. There is an urgent need for developing therapeutics to slow or potentially prevent the development of AD. In response, new therapeutic strategies and experimental drugs for AD are emerging [4-6]. Many clinical drug trials have been undertaken for AD; however, initial results have not been encouraging. Some of the issues with the clinical trial failures have recently been discussed [7-9]. Therefore, there is a need to better understand the biochemical and pathological mechanism of the disease, which in turn may shed light on reasons underlying these recent failures and guide improved drug design towards targets and clinical outcomes.

The present Perspective proposes a plausible explanation for the recent failure of an Eli Lilly BACE1 drug trial, and offers a testable model to explain the off-target effects of the drug, with a focus to learn lessons that would help prevent such failures in the future.

\section{BACE1 as a relevant target for AD?}

Neuropathologically, $\mathrm{AD}$ is characterized by the presence of amyloid- $\beta$ (A $\beta$ ) peptide plaques in the hippocampus and cerebral cortex of the brain, which provides a primary diagnostic criterion of $\mathrm{AD}$ [1]. $\mathrm{AD}$ is believed to result from the dysregulation of the production and/or turnover of $\mathrm{A} \beta$ [10]. Hence, the $\beta$-site APP-cleaving enzyme 1 (BACE1), the rate-limiting enzyme in the pathway that produces $A \beta$ peptide from the A $\beta$ precursor protein (APP) [11], is considered a promising target for the prevention or therapy of $\mathrm{AD}$ [12].

BACE1 mRNAs are transcribed from a $30.6 \mathrm{~kb}$ region of chromosome 11q23.2-11q23.3 consisting of nine exons and eight introns [13]. BACE1 genomic structure and functional characterization reveals that both the promoter region, and 5'- and 3'-untranslated regions (UTR) are subjected to regulation [14-16]. Indeed, transcriptional regulation of BACE1 by p25/cdk5 leads to enhanced amyloidogenic processing [17]. Thus, changes in the activity of the promoter region could play an important role in regulating the level of BACE1 and accompanying activity in neurons [14]. By analogy, drug-based inhibition of the enzyme may have a similar effect as regulating promoter activity (i.e., changing the overall BACE1 activity level) and prove effective in treating AD. Production of A $\beta$ from APP also involves 
the $\gamma$-secretase complex. However, inhibition of $\gamma$-secretase runs the risk of interfering in the broadly-implicated notch signaling pathway [18]. BACE1 knockout mice have not been reported to exhibit any dramatic ill effects over the course of their lifespan [19], although less attention has been paid to reports of timidity and reduced exploratory behavior that accompanies BACE1 knockout [20]. Thus, assuming the validity of the amyloid hypothesis, drug-induced inhibition of BACE1 activity would appear to be an ideal anti-AD strategy.

\section{Failure of a BACE1 inhibitor clinical trial}

Unfortunately, a recent Phase 2 trial of the LY2886721 BACE1 inhibitor from Eli Lilly may have, at least temporarily, called this anti-AD strategy into question, due to signs of liver toxicity in test subjects [21]. Eli Lilly has stated that they believe this to be consequent to a secondary effect, unrelated to the drug's mechanism of action. At first blush, this is a reasonable conclusion. After all, BACE1 knockout mice are viable and grow to adulthood without obvious liver injury [19]. Of potentially greater interest, BACE1 knockout mice have a variety of what would be presumed to be indicators of superior health, including lower fat, greater insulin sensitivity, and higher levels of brown adipose tissue [22]. However, in light of the LY2886721 trial outcome, deeper examination of BACE1 activity on substrates other than APP may indicate mechanisms that require additional attention.

\section{BACE1 catalyzes more than $A \beta$ cleavage}

\section{Implications of BACE1 off-site inhibition: Aberrant spindle formation, demyelination and impaired motor coordination}

In addition to APP processing, BACE1 plays an important role in other pathways. For example, peripheral nerves in newborn BACE1 knockout mice are thinly myelinated [23-24]. In a recent study, researchers have reported that mice require BACE1 to form and sustain muscle spindles [25]. This study demonstrated that normal spindle function was impaired not only during development but also into adulthood in BACE1 knockout animals. They showed that BACE1 deficiency in adult mice impairs motor coordination. Movement defects arise from abnormal morphology and low numbers of muscle spindles. Interestingly, alike deficiencies developed in wild-type mice treated with a structurally different BACE1 inhibitor LY2811376, a Lilly agent that lowered A $\beta$ within the cerebrospinal fluid of healthy volunteers. Lilly terminated development of this compound consequent to its toxic properties in rats [26].

Spindle formation and maturation requires BACE1 processing of neuregulin 1 (Nrg1), a transmembrane protein that regulates myelination. BACE1 and the disintegrin and metalloproteinase ADAM10 have recently been described to cleave $\mathrm{Nrg} 1$ to generate a fragment that signals in a paracrine manner and rescues myelination in BACE1 knockout zebrafish [27-28].

\section{Implications of BACE1 inhibition off-site: Liver damage or susceptibility or toxicity}

In addition to its muscle tissue and neurological activity, BACE1 is the major enzyme that cleaves $\beta$-galactoside a-2,6-sialyltransferase I (ST6Gal I) within the liver [29-30]. This generates a sialyl a-2,6-galactose residue, and the enzyme is secreted from the cell 
following proteolytic cleavage [31]. ST6Gal I cleavage is necessary for its secretion, and it is the secreted ST6Gal I that is active in glycoprotein sialylation in response to radiation stress. This protein modification is an essential step in resistance to radiation-induced cellular damage. Elimination of glycoprotein sialylation results in greater radiation-induced cytotoxicity [32]. BACE1 knockout mice have one third as much plasma ST6Gal I as control mice [30].

\section{Explaining the off-site but on-target results for BACE1 inhibitor BACE1 cleavage of APP in the brain and production of $A \beta$}

BACE1 activity on APP is a two-step process. APP protein is cleaved by BACE1, producing a secreted/soluble fragment of APP (sAPP $\beta$ ) and C99 C-terminal fragment (CTF), which is further cleaved by the $\gamma$-secretase complex to release A $\beta$ and the APP intracellular domain (AICD). The alternative APP processing pathway involves cleavage by a-secretase, producing a shorter, non-amyloidogenic P3 product, sAPPa, and C83 CTF. Notably, sAPPa is neurotrophic and may be implicated in "neuronal overgrowth" models of disorders such as autism [33]. However, APP is one of the major substrates of BACE1, among many others across different tissues.

\section{Compensatory vs. toxic model}

We propose a model for how inhibition of BACE1 might alter biology in the brain and liver based on these different threads of protein processing. In a normal human and in wild-type mice (Fig. 1A, Fig. 2A), the brain would produce physiological levels of $A \beta$ and sAPP $\beta$ by BACE1 activity. In the liver, ST6Gal I is processed by hepatic BACE1 activity to secreted ST6Gal I (sST6Gal1), which plays a vital role in resistance to cellular damage resulting from ionizing radiation exposure. This would include generation of reactive oxygen species (ROS), DNA damage, and other aberrations that are, as is general knowledge, also present as a consequence of aging. Thus by virtue of the presence of sST6Gal I in normal human (liver), the effect of ROS-mediated damage would be mitigated. In contrast, following inhibition of BACE1 activity by a small molecule inhibitor, such as Lilly compound LY2886721, in the human, we may expect that hepatic cellular damage to accumulate and result in liver injury (Fig. 1B).

Specifically, we propose that adult human hepatocytes would rely on BACE1-cleavage of ST6Gal I and the resulting sialyltransferase activity. BACE1 inhibition by small molecule inhibitors would induce a rapid reduction in BACE1 activity without adequate levels of redundant pathways in place to appropriately compensate in the human, unlike in BACE1 knockout mice (described below). These adult human cells would likely have already amassed oxidative and other age-related damage (unlike in mice with a short lifespan) that would require basal ST6GalI activity to prevent hepatocyte injury. During acute inhibition of BACE1 activity by drug treatment, the sialylated glycoprotein levels would then be depleted, leading to accumulation of cellular damage with resultant liver toxicity in human (Fig. 1B).

In the case of BACE1 knock-out mice, two factors potentially interfere with development of such injury. First, BACE1 knock-out mice begin their lives without a functional BACE1 
gene, thereby allowing time during development for actions of functionally redundant paralogues, possibly BACE2 [34] or cathepsin D [35], to compensate for the absence of BACE1 and permit apparently normal hepatic functions, albeit at reduced overall levels of ST6Gal I cleavage (Fig. 2B). Second, a mouse, BACE1 knock-out or otherwise, is highly unlikely to live long enough to properly model the decades of cumulative damage or agerelated loss of cellular protectants, such as melatonin, in human subjects. There may even be different tissue-specific trajectories in mice vs. humans [36-37]. For example, elderly human control subjects have been shown to have significantly decreased CSF melatonin levels, with an even greater decrement in $\mathrm{AD}$ patients. In contrast, aging mice have high melatonin levels in the brain [37].

Even in non-knockout mice to which LY2886721 has been administered, the very short time period between birth and administration of the drug, combined with the short duration of most animal studies and the barrier conditions in which they are maintained, may be expected to reduced underlying burden of accumulated cellular damage compared to adult human subjects, such that inhibition of cleavage of STGal6 I by BACE1 may not have discernable toxic effects on the liver. We propose that acute global inhibition of BACE1 activity in adult humans, on the other hand, may result in apparent liver toxicity due to disrupted production of indispensable detoxification and cellular repair activities of sialylated glycoproteins (Fig. 1B). Lilly has yet to publish results of the actions of LY2886721 on ST6Gal I processing in animals or human plasma that would indicate whether this proposed model might explain the hepatotoxicity observed in their phase 2 trial.

\section{Evidence for the off-site but on-target effects of BACE1}

We have examined known expression patterns, available from the Human Protein Atlas [38], for BACE1, APP and ST6Gal I. Normal tissue expression levels were cross-indexed for these proteins, and tissues in which expression was ranked at "Moderate" or "High" (on a scale of "None" < "Low" < "Moderate" < "High") for BACE1 and APP or ST6Gal I were recorded. In addition, this analysis was performed substituting BACE2 and CTSD (Table 1). Interestingly, we noticed that there is very little overlap between BACE1+APP and BACE1+ST6Gal I cross-indexed expression, with it only occurring in the glandular cells of the epididymis and the adrenal glands. Notably, this pattern is closely matched with BACE2 or CTSD. This supports the notion that on-target inhibition of BACE1 could have highly disparate effects in different organ systems, including those sites where targeting was not intended.

\section{Alternative explanation for unwanted effects of BACE1 inhibition}

We recognize the fact that drug-induced liver toxicity is the most common cause of hepatic dysfunction, of drug failures during clinical trials and post-market withdrawal of an approved drug [39], and is usually due to the chemical structure of a particular experimental drug rather than the therapeutic target.

A primary function of the liver is to metabolize foreign compounds and hence it is often the principal site of exposure to toxins. Hepatic metabolism of drugs involves their biotransformation to facilitate their elimination, with the majority of chemical modifications 
being red-ox reactions, catalyzed by $\mathrm{P} 450$ enzymes (Phase I), and conjugation ones with endogenous agents (glutathione, glucuronate or sulfate: Phase II) to generate non-toxic metabolites that can be excreted in urine or bile. Albeit considered a detoxification process, drug biotransformation of specific chemical structures can result in bioactivation and, by various mechanisms, induce hepatocyte injury [40]. Comprehensive analyses of bioactivation pathways of organic functional groups are available [41]. As examples among many, quinones may potentially cause toxicity via ROS formation [42], as may quinone imines (generated by the twoelectron oxidation of ortho - and para -aminophenols) [43] exemplified by the metabolism of acetaminophen, indomethicin and the anti-malerial drug amodiaquine that can result in dosedependent hepatotoxicity and, for the latter, other toxicities too [40]. Hence, a balance between detoxification, bioactivation and a host of defense/repair mechanisms ultimately determines whether or not a drug will elicit a toxic effect. Consequently, the relationship between bioactivation and hepatotoxicity is a far from simple one [44-45] and can result in drug withdrawal or a FDA boxed warning when toxicity occurs at therapeutic doses, or a lack of hepatotoxicity as occurs with therapeutic doses of acetaminophen - but overdose leading to hepatocyte dysfunction and death [46].

Therefore, our Perspective assumes significance by proposing an alternative model of liver toxicity, based on a scientific hypothesis that is testable and capable to stimulate further relevant research.

In addition to our hypothesis, alternative explanations are also worth evaluating. For example, one could invoke idiosyncratic drug-induced liver injury (IDILI). Recentlypublished reviews place its frequency at 19 per 100,000 treated individual [47], and IDILI can manifest in multiple fashions. It accounts for up to $13 \%$ of acute liver failure in the United States. IDILI is unpredictable in nature, has a poorly understood pathogenesis, but several factors including genetic, epigenetic, and metabolism variation are known to contribute to its likelihood [47-49]. In general, it results in the over/under expression of drug metabolizing enzymes, a different drug metabolism pattern, and ultimately in the abnormal generation of a toxic metabolite that has dose-dependent action in vulnerable individuals [50]. IDILI can additionally be elicited by the induction of an autoimmune response by commercial pharmaceuticals or traditional "herbal" treatments [50-51]. However, their relative rarity means that they usually escape being detected in preclinical assessments and clinical trials [50]. Currently available data from the Lilly study does not answer which, if either, of these hypotheses actually explains the observed liver damage. From the viewpoint of commercial success, an idiosyncratic event would be highly preferable to a previously unconsidered off-site drug effect. However, it is reasonable to keep in mind testing each of them in turn, if necessary, and pre-excluding neither.

\section{Alternative therapeutic strategies for the on-site effect of BACE1 inhibition}

If liver injury is, indeed, a consequence of acute global reduction in BACE1 activity, alternative therapeutic strategies would be required to circumvent this problem. BACE1 inhibitors that selectively target the CNS are one possibility, although the technology to achieve this has yet to be developed. Therapeutic windows may exist for some small molecule inhibitors for which moderate global inhibition of BACE1 may be found to be 
clinically effective without inducing liver injury. Strategies to selectively inhibit BACE1 expression levels represent an additional possibility. Exogenous delivery of siRNA directed against BACE1 is one example worth exploring. However, intravenously delivered siRNA are generally sequestered by the liver, spleen, kidney, pancreas and bone marrow [52], potentially exacerbating hepatotoxic effects of BACE1 knockdown. However, preclinical studies have also demonstrated that BACE1 siRNA can be selectively targeted to the CNS using RVG-tagged exosomal delivery [53].

Exogenous delivery of microRNA (miRNA) is an alternative possibility. MiRNA are endogenously expressed 18-26 nucleotides small RNA species that predominantly act to downregulate expression of target transcripts [54]. Furthermore, several miRNAs have been shown to be implicated in cancer and neurodegenerative disorders [55-56]. Indeed, specific miRNAs, such as miR-101 and miR-153, regulate APP expression and are dysfunctional in AD brain [57-58]. Notably, miR-339-3p has recently been shown to regulate BACE1 expression in human brain cultures [59]. Interestingly, endogenous expression of miRNA varies among different cell types and tissues. The endogenous expression pattern of miRNA could be exploited to selectively reduce BACE1 expression in the CNS. Systemic delivery of a BACE1-targeting miRNA would be expected to have a more dramatic effect on BACE1 expression in tissues with naturally low endogenous expression levels. Therefore, a BACE1targeting miRNA with high endogenous levels in the liver and low endogenous levels in the brain would be expected to have a more potent inhibiting effect on brain BACE1 expression, particularly if localized delivery was accomplished (potentially intra-nasally).

\section{Limitations of data from animal models and Closing Remarks}

The failed trial of LY2886721 illustrates the limitations of data from animal models when evaluating the actions of acute enzyme inhibition in older human subjects. Indeed, caution should be used when testing the suitability of drug candidates for AD using animal model paradigms, such as knockout mice or short-term drug treatment designs. The short lifespan of these animals maintained in barrier conditions could mask possible toxic effects of drugs that would only reveal themselves when tested in human subjects with AD that has developed over decades with the influence of environment, diet and lifestyle playing an important role [60-61]. We do not claim that our proposed model necessarily explains the setback in the LY2886721 trial, but it does suggest that it may be imprudent to dismiss, so confidently, primary drug activity out-of-hand as a problematic factor. We should point out that our model and opinion are based only on the published literature and available data; and unfortunately, we do not have access to confidential Lilly safety data either from the Company or FDA.

Indeed, our hypothesis equally applies to other BACE1/2 inhibitors, either completed, contemplated or ongoing. We have taken merely the Lilly compound as an example, which passed Phase I but not the Phase II clinical trials, the latter generally being of substantially longer duration. Merck \& Co has recently presented positive clinical results with its BACE inhibitor for $\mathrm{AD}$ [62]. The data was early stage arising from a phase $\mathrm{Ib}$ (7 day) study. It confirmed that MK-8931 reduced cerebrospinal fluid (CSF) levels of $A \beta$ in patients with mild-to-moderate AD. Merck has also started a larger phase II/III study of MK-8931 in AD 
patients - called EPOCH (to enroll up to 1960 subjects with a treatment duration of up to 78 weeks [63]), with the potential to expand into pivotal trial if early safety and efficacy objectives are met. Other BACE inhibitors coming through the clinical pipeline for AD include Eisai's E2609, Roche's RG7129 and AstraZeneca's AZD3293, all of which are in early-stage evaluation. We are uncertain whether these Companies, which are currently engaged in multiple-dose clinical trials, are testing their drugs in our alternative model described herein, including the activity measurement of STGal6 I in liver.

In summary, our proposed "Compensatory vs. toxic model" also suggests therapeutic alternatives by which toxicity could potentially be excluded. Likewise many drugs' so called "off-target" effects could be checked for "off-site" effects, which are not necessarily offtarget. A similar caution should be taken in using genetic animal models, such as knockout mice, and/or short-term treatment in short-lived mice, as they may not reveal the toxic effects of drugs tested in human with $\mathrm{AD}$ or an alike disease that takes over six decades to develop with environment, diet and lifestyle playing important roles.

In synopsis, we encourage the evaluation of BACE1/2 inhibitors for not only on-target actions (such as BACE1 inhibition in the CNS) but also non-structural off-targets ones that are not necessarily off-BACE (such as BACE1 inhibition on STGal6 I and other substrates in liver and other tissues). The message is not only a lesson learnt from the recent clinical trial failures but also its application to the on-going trials.

\section{Acknowledgments}

We sincerely appreciate the grant support from the Alzheimer's Association (Zenith award and IIRG) and from the NIH (grant \# AG18379, AG18884 and AG042804) to DKL.

\section{References}

1. Thies W, Bleiler L. 2013 Alzheimer's disease facts and figures. Alzheimers Dement. 2013; 9:20845. [PubMed: 23507120]

2. Wu J, Basha MR, Brock B, Cox DP, Cardozo-Pelaez F, McPherson CA, Harry J, Rice DC, Maloney B, Chen D, Lahiri DK, Zawia NH. Alzheimer's disease (AD)-like pathology in aged monkeys after infantile exposure to environmental metal lead $(\mathrm{Pb})$ : evidence for a developmental origin and environmental link for AD. J Neurosci. 2008; 28:3-9. [PubMed: 18171917]

3. Lahiri DK, Maloney B, Zawia NH. The LEARn model: an epigenetic explanation for idiopathic neurobiological diseases. Mol Psychiatry. 2009; 14:992-03. [PubMed: 19851280]

4. Corbett A, Ballard C. New and emerging treatments for Alzheimer's disease. Expert Opin Emerg Drugs. 2012; 17:147-56. [PubMed: 22439621]

5. Huang Y, Mucke L. Alzheimer mechanisms and therapeutic strategies. Cell. 2012; 148:1204-22. [PubMed: 22424230]

6. Bailey JA, Ray B, Greig NH, Lahiri DK. Rivastigmine lowers Abeta and increases sAPPalpha levels, which parallel elevated synaptic markers and metabolic activity in degenerating primary rat neurons. PLoS One. 2011; 6:e21954. [PubMed: 21799757]

7. Schneider LS, Lahiri DK. The perils of Alzheimer's drug development. Curr Alzheimer Res. 2009; 6:77-8. [PubMed: 19199878]

8. Becker RE, Greig NH. Why so few drugs for Alzheimer's disease? Are methods failing drugs? Curr Alzheimer Res. 2010; 7:642-51. 
9. Sambamurti K, Greig NH, Utsuki T, Barnwell EL, Sharma E, Mazell C, et al. Targets for AD treatment: conflicting messages from gamma-secretase inhibitors. J Neurochem. 2011; 117:359-74. [PubMed: 21320126]

10. Hardy J. The amyloid hypothesis for Alzheimer's disease: a critical reappraisal. J Neurochem. 2009; 110:1129-34. [PubMed: 19457065]

11. Vassar R, Bennett BD, Babu-Khan S, Kahn S, Mendiaz EA, Denis P, et al. Beta-secretase cleavage of Alzheimer's amyloid precursor protein by the transmembrane aspartic protease BACE. Science. 1999; 286:735-41. [PubMed: 10531052]

12. Li Y, Zhou W, Tong Y, He G, Song W. Control of APP processing and Abeta generation level by BACE1 enzymatic activity and transcription. FASEB J. 2006; 20:285-92. [PubMed: 16449801]

13. Sambamurti K, Kinsey R, Maloney B, Ge YW, Lahiri DK. Gene structure and organization of the human beta-secretase (BACE) promoter. FASEB J. 2004; 18:1034-6. [PubMed: 15059975]

14. Ge YW, Maloney B, Sambamurti K, Lahiri DK. Functional characterization of the 5' flanking region of the BACE gene: identification of a $91 \mathrm{bp}$ fragment involved in basal level of BACE promoter expression. FASEB J. 2004; 18:1037-39. [PubMed: 15059977]

15. Lahiri DK, Ge YW, Rogers JT, Sambamurti K, Greig NH, Maloney B. Taking down the unindicted co-conspirators of amyloid beta-peptide-mediated neuronal death: shared gene regulation of BACE1 and APP genes interacting with CREB, Fe65 and YY1 transcription factors. Curr Alzheimer Res. 2006; 3:475-83. [PubMed: 17168646]

16. Lahiri DK, Maloney B, Ge YW. BACE1 gene promoter is differentially regulated: Detection of a novel promoter region for its cell type-specific regulation. J Mol Neurosci. 2006; 28:193-210. [PubMed: 16679558]

17. Wen Y, Yu WH, Maloney B, Bailey J, Ma J, Marie I, et al. Transcriptional regulation of betasecretase by p25/cdk5 leads to enhanced amyloidogenic processing. Neuron. 2008; 57:680-90. [PubMed: 18341989]

18. Imbimbo BP, Panza F, Frisardi V, Solfrizzi V, D'Onofrio G, Logroscino G, et al. Therapeutic intervention for Alzheimer's disease with gamma-secretase inhibitors: still a viable option? Expert Opin Investig Drugs. 2011; 20:325-41.

19. Luo Y, Bolon B, Kahn S, Bennett BD, Babu-Khan S, Denis P, et al. Mice deficient in BACE1, the Alzheimer's beta-secretase, have normal phenotype and abolished betaamyloid generation. Nat Neurosci. 2001; 4:231-32. [PubMed: 11224535]

20. Harrison SM, Harper AJ, Hawkins J, Duddy G, Grau E, Pugh PL, et al. BACE1 (beta secretase) transgenic and knockout mice: identification of neurochemical deficits and behavioral changes. Mol Cell Neurosci. 2003; 24:646-55. [PubMed: 14664815]

21. Rogers, MB. Lilly Halts Phase 2 Trial of BACE Inhibitor Due to Liver Toxicity. Alzheimer Research Forum. 2013. http://www.alzforum.org/new/detail.asp?id=3522

22. Meakin PJ, Harper AJ, Hamilton DL, Gallagher J, McNeilly AD, Burgess LA, et al. Reduction in BACE1 decreases body weight, protects against diet-induced obesity and enhances insulin sensitivity in mice. Biochem J. 2012; 441:285-96. [PubMed: 21880018]

23. Willem M, Garratt AN, Novak B, Citron M, Kaufmann S, Rittger A, et al. Control of peripheral nerve myelination by the beta-secretase BACE1. Science. 2006; 314:664-6. [PubMed: 16990514]

24. Hu X, Hicks CW, He W, Wong P, Macklin WB, Trapp BD, et al. Bacel modulates myelination in the central and peripheral nervous system. Nat Neurosci. 2006; 9:1520-5. [PubMed: 17099708]

25. Cheret C, Willem M, Fricker FR, Wende H, Wulf-Goldenberg A, Tahirovic S, et al. Bace1 and Neuregulin-1 cooperate to control formation and maintenance of muscle spindles. EMBO J. 2013; 32:2015-28. [PubMed: 23792428]

26. May PC, Dean RA, Lowe SL, Martenyi F, Sheehan SM, Boggs LN, et al. Robust central reduction of amyloid-beta in humans with an orally available, non-peptidic beta-secretase inhibitor. $\mathrm{J}$ Neurosci. 2011; 31:16507-16. [PubMed: 22090477]

27. Fleck D, van Bebber F, Colombo A, Galante C, Schwenk BM, Rabe L, et al. Dual cleavage of neuregulin 1 type III by BACE1 and ADAM17 liberates its EGF-like domain and allows paracrine signaling. J Neurosci. 2013; 33:7856-69. [PubMed: 23637177]

28. van Bebber F, Hruscha A, Willem M, Schmid B, Haass C. Loss of Bace2 in zebrafish affects melanocyte migration and is distinct from Bace1 knock out phenotypes. J Neurochem. 2013 
29. Kitazume S, Oka R, Ogawa K, Futakawa S, Hagiwara Y, Takikawa H, et al. Molecular insights into beta-galactoside alpha2,6-sialyltransferase secretion in vivo. Glycobiology. 2009; 19:479-87. [PubMed: 19150807]

30. Kitazume S, Nakagawa K, Oka R, Tachida Y, Ogawa K, Luo Y, et al. In vivo cleavage of alpha2,6-sialyltransferase by Alzheimer beta-secretase. J Biol Chem. 2005; 280:8589-95. [PubMed: 15364953]

31. Kitazume S, Tachida Y, Oka R, Shirotani K, Saido TC, Hashimoto Y. Alzheimer's beta-secretase, beta-site amyloid precursor protein-cleaving enzyme, is responsible for cleavage secretion of a Golgi-resident sialyltransferase. Proc Natl Acad Sci USA. 2001; 98:13554-9. [PubMed: 11698669]

32. Lee M, Lee HJ, Bae S, Lee YS. Protein sialylation by sialyltransferase involves radiation resistance. Mol Cancer Res. 2008; 6:1316-25. [PubMed: 18708363]

33. Lahiri DK, Sokol DK, Erickson C, Ray B, Ho CY, Maloney B. Autism as early neurodevelopmental disorder: evidence for an sAPPalpha-mediated anabolic pathway. Front Cell Neurosci. 2013; 7:94. [PubMed: 23801940]

34. Solans A, Estivill X, de La Luna S. A new aspartyl protease on 21q22.3, BACE2, is highly similar to Alzheimer's amyloid precursor protein beta-secretase. Cytogenetics and cell genetics. 2000; 89:177-84. [PubMed: 10965118]

35. Schechter I, Ziv E. Kinetic properties of cathepsin D and BACE 1 indicate the need to search for additional beta-secretase candidate(s). Biol Chem. 2008; 389:313-20. [PubMed: 18177262]

36. Liu RY, Zhou JN, van Heerikhuize J, Hofman MA, Swaab DF. Decreased melatonin levels in postmortem cerebrospinal fluid in relation to aging, Alzheimer's disease, and apolipoprotein Eepsilon4/4 genotype. J Clin Endrocrinol Metab. 1999; 84:323-7.

37. Lahiri DK, Ge YW, Sharman EH, Bondy SC. Age-related changes in serum melatonin in mice: higher levels of combined melatonin and 6-hydroxymelatonin sulfate in the cerebral cortex than serum, heart, liver and kidney tissues. J Pineal Res. 2004; 36:217-23. [PubMed: 15066045]

38. Ponten F, Jirstrom K, Uhlen M. The Human Protein Atlas--a tool for pathology. J Pathol. 2008; 216:387-93. [PubMed: 18853439]

39. Norris W, Paredes AH, Lewis JH. Drug-induced liver injury in 2007. Curr Opin Gastroenterol. 2008; 24:287-97. [PubMed: 18408456]

40. Leung L, Kalgutkar AS, Obach RS. Metabolic activation in drug-induced liver injury. Drug Metab Rev. 2012; 44:18-33. [PubMed: 21939431]

41. Kalgutkar AS, Gardner I, Obach RS, Shaffer CL, Callegari E, Henne KR, Mutlib AE, Dalvie DK, Lee JS, Nakai Y, O'Donnell JP, Boer J, Harriman SP. A comprehensive listing of bioactivation pathways of organic functional groups. Curr Drug Metab. 2005; 6:161-225. [PubMed: 15975040]

42. Bolton JL, Trush MA, Penning TM, Dryhurst G, Monks TJ. Role of quinones in toxicology. Chem Res Toxicol. 2000; 13:135-160. [PubMed: 10725110]

43. Monks TJ, Jones DC. The metabolism and toxicity of quinones, quinonimines, quinone methides, and quinonethioethers. Curr Drug Metab. 2002; 3:425-438. [PubMed: 12093358]

44. Williams DP. Toxicophores: investigations in drug safety. Toxicology. 2006; 226:1-11. [PubMed: 16860917]

45. Johnson WW. Many drugs and phytochemicals can be activated to biological reactive intermediates. Curr Drug Metab. 2008; 9:344-51. [PubMed: 18473753]

46. Jaeschke H, Bajt ML. Intracellular signaling mechanisms of acetaminophen-induced liver cell death. Toxicol Sci. 2006; 89:31-41. [PubMed: 16177235]

47. Hussaini SH, Farrington EA. Idiosyncratic drug-induced liver injury: an update on the 2007 overview. Expert Opin Drug Saf. 2013

48. Corsini A, Ganey P, Ju C, Kaplowitz N, Pessayre D, Roth R, et al. Current challenges and controversies in drug-induced liver injury. Drug Saf. 2012; 35:1099-117. [PubMed: 23137150]

49. Vinken M, Maes M, Vanhaecke T, Rogiers V. Drug-induced liver injury: mechanisms, types and biomarkers. Current medicinal chemistry. 2013; 20:3011-21. [PubMed: 23746274]

50. Cardenas A, Restrepo JC, Sierra F, Correa G. Acute hepatitis due to shen-min: a herbal product derived from Polygonum multiflorum. J Clin Gastroenterol. 2006; 40:629-32. [PubMed: 16917407] 
51. Njoku DB. Suppressive and pro-inflammatory roles for IL-4 in the pathogenesis of experimental drug-induced liver injury: a review. Expert Opin Drug Metab Toxicol. 2010; 6:519-31. [PubMed: 20166853]

52. Larson SD, Jackson LN, Chen LA, Rychahou PG, Evers BM. Effectiveness of siRNA uptake in target tissues by various delivery methods. Surgery. 2007; 142:262-9. [PubMed: 17689694]

53. Alvarez-Erviti L, Seow Y, Yin H, Betts C, Lakhal S, Wood MJ. Delivery of siRNA to the mouse brain by systemic injection of targeted exosomes. Nat Biotechnol. 2011; 29:341-5. [PubMed: 21423189]

54. Bartel DP. MicroRNAs: target recognition and regulatory functions. Cell. 2009; 136:215-33. [PubMed: 19167326]

55. Long JM, Lahiri DK. Advances in MicroRNA experimental approaches to study physiological regulation of gene products implicated in CNS disorders. Exp Neurol. 2012

56. Holohan KN, Lahiri DK, Schneider BP, Foroud T, Saykin AJ. Functional microRNAs in Alzheimer's disease and cancer: differential regulation of common mechanisms and pathway. Front Genet. 2012; 3:323. [PubMed: 23335942]

57. Long JM, Lahiri DK. MicroRNA-101 downregulates Alzheimer's amyloid-beta precursor protein levels in human cell cultures and is differentially expressed. Biochem Biophys Res Commun. 2011; 404:889-95. [PubMed: 21172309]

58. Long JM, Ray B, Lahiri DK. MicroRNA-153 physiologically inhibits expression of amyloid-beta precursor protein in cultured human fetal brain cells and is dysregulated in a subset of Alzheimer disease patients. J Biol Chem. 2012; 287:31298-310. [PubMed: 22733824]

59. Long JM, Ray B, Lahiri DK. Human microRNA-339-5p negatively regulates $\beta$-site amyloid precursor protein cleaving enzyme 1 (BACE1) in human primary brain cultures and is dysregulated in a subset of Alzheimer's disease subjects. Submitted to J Biol Chem. 2013

60. Lahiri DK, Maloney B, Basha MR, Ge YW, Zawia NH. How and when environmental agents and dietary factors affect the course of Alzheimer's disease: the "LEARn"model (latent early-life associated regulation) may explain the triggering of AD. Curr Alzheimer Res. 2007; 4:219-28. [PubMed: 17430250]

61. Lahiri DK. Prions: a piece of the puzzle? Science. 2012; 337:1172. [PubMed: 22955815]

62. http://www.mercknewsroom.com/press-release/alzheimers-disease/merck-presentsfindingsphase-1b-study-investigational-bace-inhibit (viewed October 28, 2013)

63. http://clinicaltrials.gov/show/NCT01739348 (viewed October 28, 2013) 

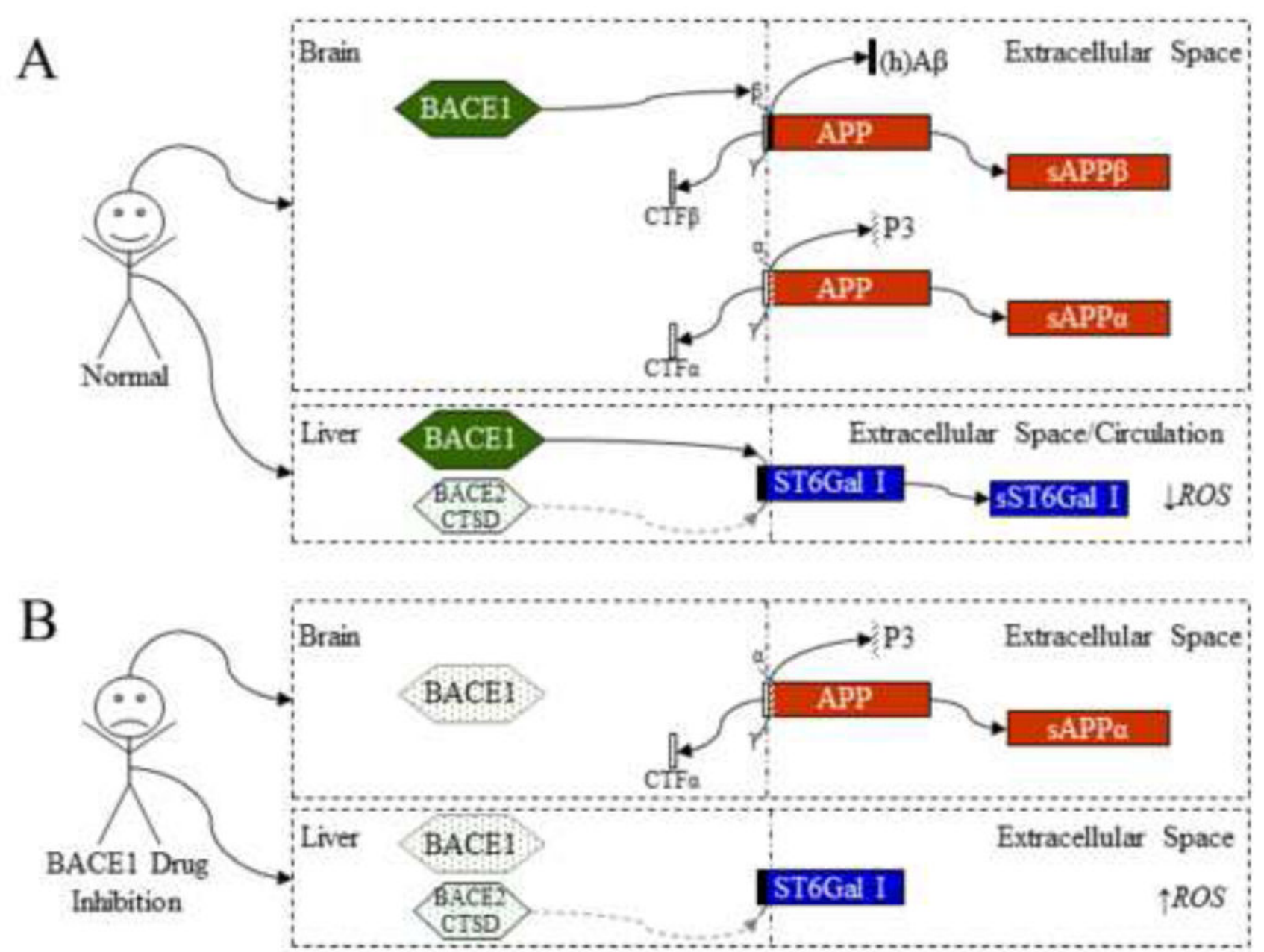

Fig. 1. Effects of Altering BACE1 ( $\beta$-Secretase) Activities in Human Brain and Liver by Drug Inhibition

A) Normal Human BACE1 activity. BACE1 in brain processes amyloid precursor protein (APP, red) at the $\beta$-cleavage site, producing a secreted/soluble form of APP, sAPP $\beta$. This is followed by cleavage in the cell membrane by $\gamma$-secretase complex to produce $A \beta$ (black) and $\beta \mathrm{CTF}$ (white). The predominant APP processing pathway is, however, by a-secretase, which produces sAPPa, P3 fragment, and aCTF. In liver, BACE1 is the primary enzyme catalyzing cleavage of $\beta$ galactoside a2,6-sialyltransferase (ST6Gal I, blue) into secreted ST6GAL I (sST6Gal I) and other products. The sST6Gal I sialylates appropriate proteins, resulting in reduction of reactive oxidizing species (ROS) and other age and environmentrelated damage. ROS/environmental damage need not be extracellular to be effected by sialylated proteins. Paralogous activity from BACE2 or CTSD (stippled light green), while present, would be directed towards other physiological processes. $\underline{B}$ ) Effects of sudden organism-wide reduction of BACE1 activity in adult humans. Drugs administered late in life should immediately reduce BACE1 activity in both brain and liver. In brain, BACE1 processing of APP becomes sufficiently infrequent that little to no A $\beta$ or sAPP $\beta$ are 
produced, while a-secretase is unchanged. In liver, sudden depletion of BACE1 enzymatic activity results in severe reduction or even loss of ST6Gal I processing, ultimately combining with accumulated environmental damage to produce an apparently "toxic" result. The loss of BACE1 activity is not properly compensated for by potential paralogues due to the abrupt nature of BACE1 inhibition. 

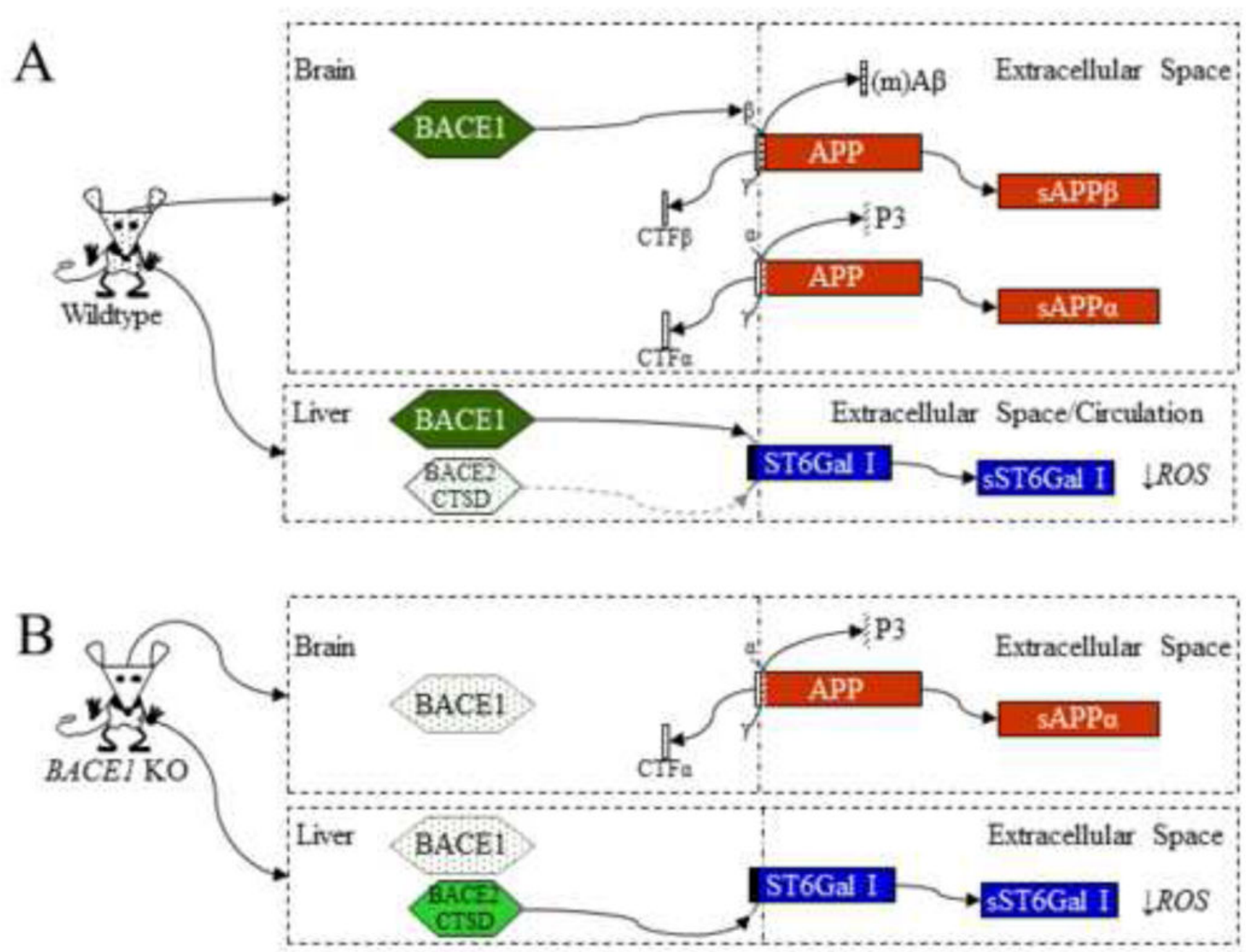

Fig. 2. Effects of BACE1 Knockout on BACE1 $\beta$-Secretase Activities in Mouse Brain and Liver A) Wild-type mouse BACE1 activity. BACE1 (green) in brain processes amyloid precursor protein (APP, red) at the $\beta$-cleavage site, producing a secreted/soluble form of APP, sAPP $\beta$. This is followed by cleavage in the cell membrane by $\gamma$-secretase complex to produce $A \beta$ (horizontal line fill—wild-type mouse $\mathrm{A} \beta$ differs from human) and $\beta \mathrm{CTF}$ (white). The predominant APP processing pathway in mice is, however, by a-secretase, which produces sAPPa, P3 fragment (gray stipple), and aCTF. In liver, BACE1 is the primary enzyme catalyzing cleavage of $\beta$ galactoside (ST6Gal I, blue) into secreted ST6GAL I (sST6Gal I) and other products. The sST6Gal I sialylates appropriate proteins, resulting in reduction of reactive oxidizing species (ROS) and other age and environment-related damage. ROS/ environmental damage need not be extracellular to be effected by sialylated proteins. Paralogous activity from BACE2 or CTSD (stippled light green), while present, would be directed towards other physiological processes. $\underline{B}$ ) Effects of lack of BACE1 activity in knockout mice. In BACE1 knockout mice, $\beta$-secretase activity in brain is lacking. No A $\beta$ is produced from processing of APP. The a-secretase pathway would still function along with $\gamma$-secretase to produce sAPPa, P3, and aCTF. However, given the absence of BACE1 during the plastic period of embryonic development, compensatory upregulation of 
paralogues such as BACE2 or CTSD (solid light green) occurs leading to sufficient cleavage of ST6Gal I (up to one third of normal total activity) to allow for normal hepatic function. Given the compensation and short lifespan, the knockout mouse shows no apparent toxicity due to unremediated environmental effects. 


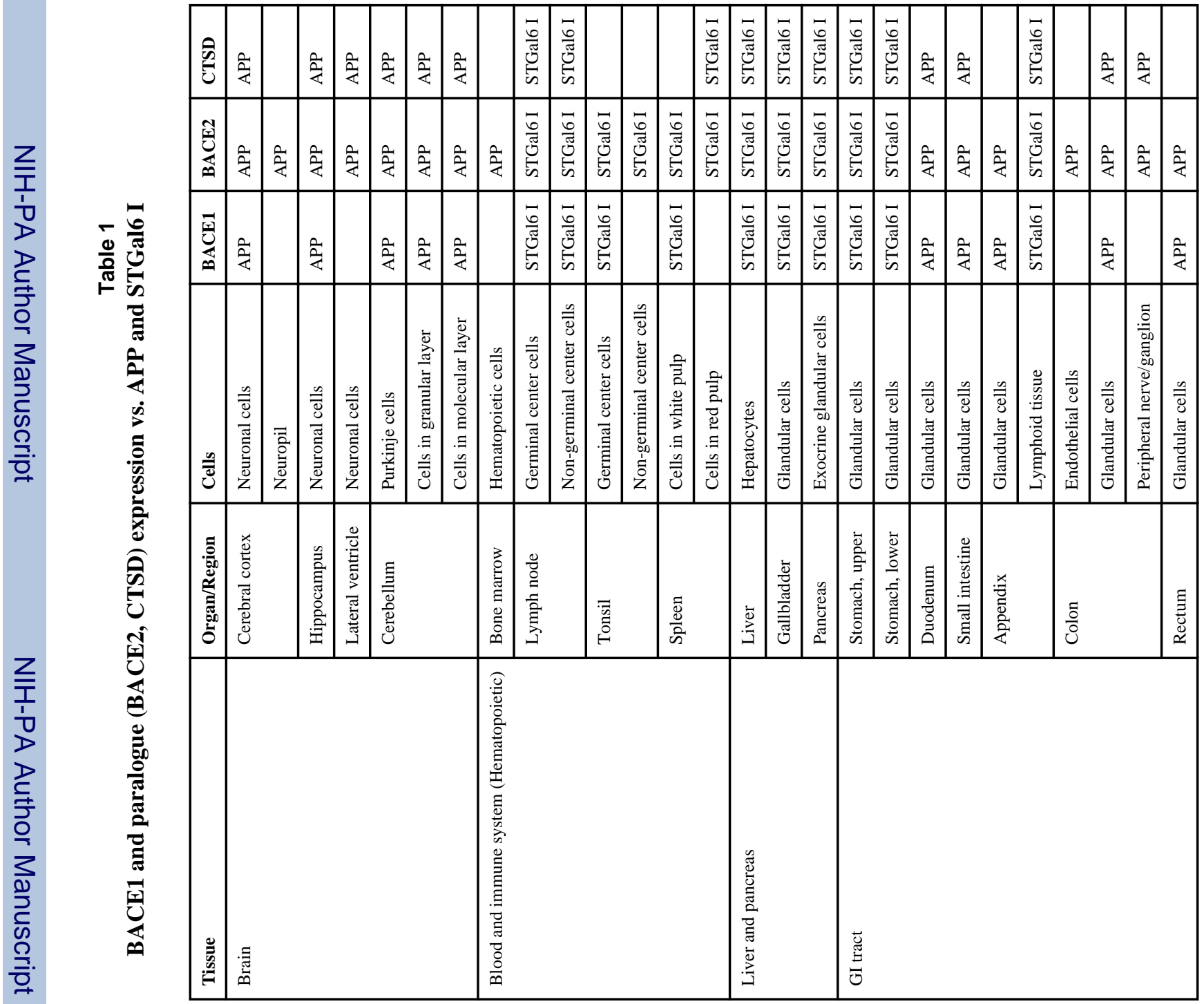




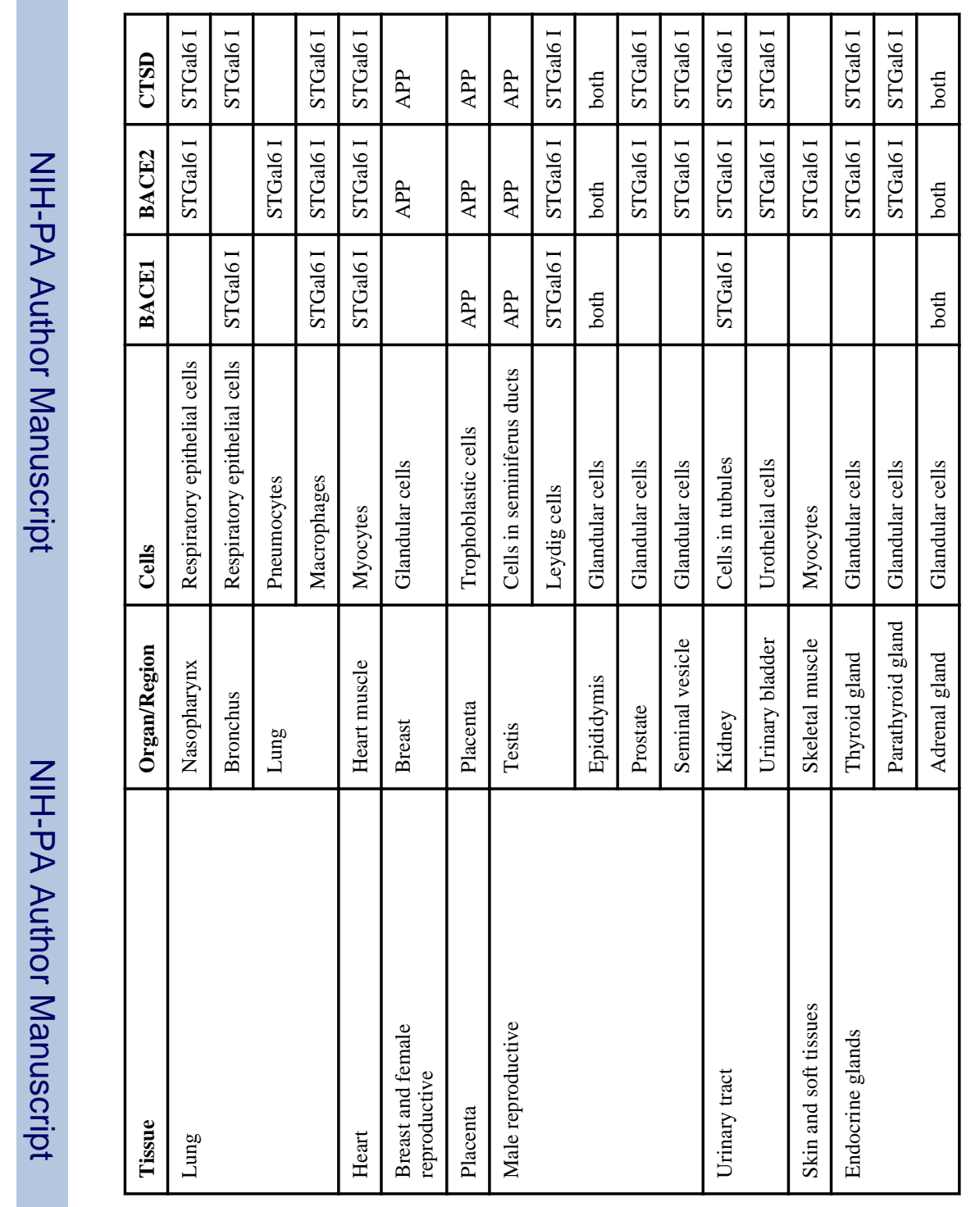

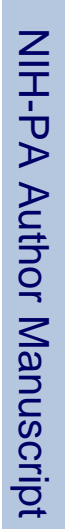

